\title{
P1780
}

\section{Usefulness of multimodality imaging approach in the diagnosis of mechanical prosthetic valve dysfunction}

L Fusini, M Muratori, G Teruzzi, S Ghulam Ali, E Innocenti, N Corrieri, G Tamborini, M Mapelli, F Alamanni, P Montorsi ... Show more

European Heart Journal, Volume 40, Issue Supplement_1, October 2019, ehz748.0532, https://doi.org/10.1093/eurheartj/ehz748.0532

Published: 21 October 2019

\begin{abstract}
Background

Although the long-term outcome of mechanical mitral and aortic prosthetic valve (M-PV, Ao-PV), PV dysfunction (PVD) remains a very serious complication associated with high morbidity and mortality. Thrombosis/pannus and paravalvular leak are the 2 main mechanisms of PVD. The diagnosis of PVD, based on clinical presentation may be challenging, but it is essential for referring the patient to the optimal treatment (clinical follow-up, thrombolysis, surgery). An integrated multimodality imaging approach, comprising several parameters by transthoracic echocardiography (TTE) and fluoroscopy (F), is mandatory to pursue the correct therapeutic pathway.
\end{abstract}

\section{Purpose}

This study aims to evaluate the incremental diagnostic value of combined TTE $+\mathrm{F}$ over each imaging modality alone in symptomatic pts with Ao-PV or M-PV and high suspicion of PVD.

\section{Methods}

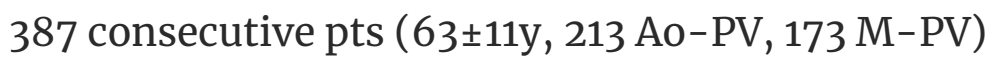
suspected for PVD, symptomatic for dyspnea, embolic events, fever or haemolysis were enrolled. All patients were imaged by TTE and F within 2 days after the 
admission to the hospital. TTE was defined positive for PVD in presence of intra/para-prosthetic regurgitation or high transprosthetic gradient ( $>20 \mathrm{mmHg}$ in Ao-PV, $>8 \mathrm{mmHg}$ in $\mathrm{M}-\mathrm{PV}$ ) together with altered Doppler parameters (for Ao-PV: DVI <0.25, AT>95ms; for M-PV: Peak Mitral Velocity >2m/sec, VTIPrMV/VTILVO>2.5, PHT $>130 m s)$. F was defined positive for PVD when leaflet/s restriction occurs. PVD was confirmed by transoesophageal echocardiography (TOE) or positive response of thrombolysis $(\mathrm{T})$, or surgical inspection (S).

\section{Results}

PVD was found in 46\% (99/213) of Ao-PV and in 53\% (91/173) of M-PV at TOE/T/S. Sensitivity (SE), specificity (SP), negative predictive value (NPV), positive predictive value (PPV) and diagnostic accuracy (ACC) for TTE, F and combined $\mathrm{TTE}+\mathrm{F}$ are reported in Table. The integration of $\mathrm{TTE}+\mathrm{F}$ data significantly improved ACC both for Ao-PV and M-PV. At ROC analysis, the combined model of TTE+F showed the highest AUC for the detection of PVD compared with TTE and F alone (Figure).

Table 1. Comparison of diagnostic accuracy between TTE, F, and TTE+F

\begin{tabular}{|c|c|c|c|c|c|c|}
\hline & $\begin{array}{l}\text { TTE- } \\
\text { Ao-PV } \\
(n=211)\end{array}$ & $\begin{array}{l}\text { F- } \\
\text { Ao_PV } \\
(n=204)\end{array}$ & $\begin{array}{l}\text { TTE+F- } \\
\text { Ao-PV } \\
(n=202)\end{array}$ & $\begin{array}{l}\text { TTE-M- } \\
\text { PV } \\
(n=172)\end{array}$ & $\begin{array}{l}\text { F-M-PV } \\
(n=158)\end{array}$ & $\begin{array}{l}\text { TTE- } \\
\text { M-PI } \\
(n=1\end{array}$ \\
\hline SE / & 86 / 89 / & 59 / 99 / & 94 / 88 / & 74 / 90 / & 49 / 96 / & $81 / i$ \\
\hline SP / & 88 / 88 / & 72 / 98 / & 94 / 88 / & 75 / 89 / & 60 / 93 / & $78 /$ \\
\hline NPV & 88 & 79 & 91 & 81 & 70 & 83 \\
\hline \multicolumn{7}{|c|}{ 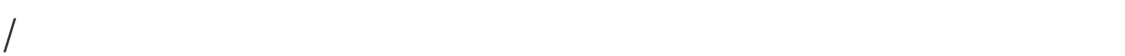 } \\
\hline \multicolumn{7}{|l|}{ PPV } \\
\hline \multicolumn{7}{|l|}{ / } \\
\hline $\mathrm{ACC}$ & & & & & & \\
\hline
\end{tabular}

$(\%)$ 
Aortic Prosthesis

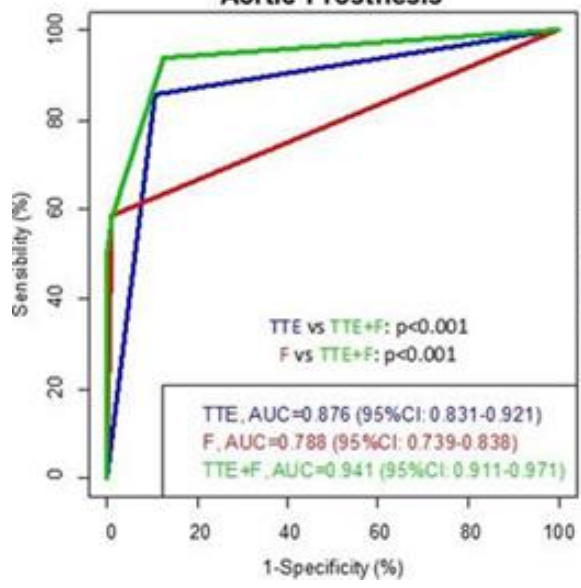

Mitral Prosthesis

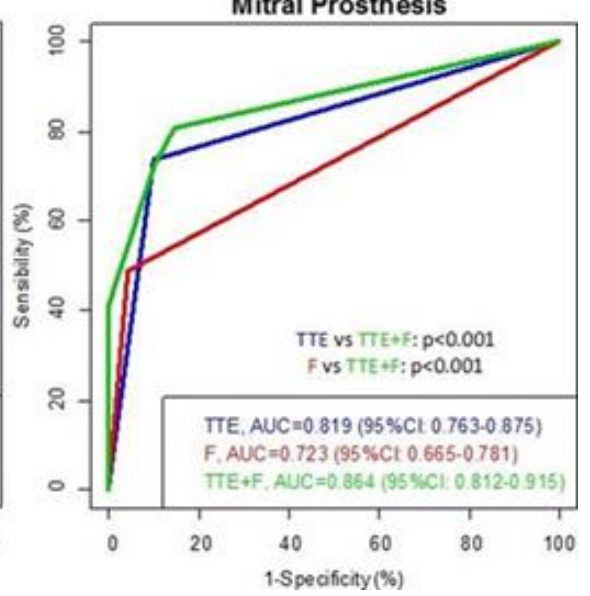

Figure 1. ROC curves

\section{Conclusions}

In patients with clinical suspicion of PVD, TTE and $\mathrm{F}$ are both valid tools to evaluate the PV performance. However, the combined model of TTE+F had a significant incremental value over TTE or $\mathrm{F}$ alone to diagnose the presence of PVD. This multimodality imaging approach allows to overcome several weaknesses of the TTE or F alone and consequently provides a prompt recognition of PVD even though TOE remains the gold standard to diagnose paravalvular Leak and non-obstructive thrombosis.

\section{Keywords: Prosthetic Heart Valves}

Topic: aorta, heart valve prosthesis, transesophageal echocardiography, hemolysis, thrombosis, thrombolytic therapy, patient referral, dyspnea, mitral valve prosthetic malfunction, fever, brachial plexus neuritis, fluoroscopy, followup, roc curve, surgical procedures, operative, toes, vomiting, diagnosis, diagnostic imaging, morbidity, mortality, embolism, echocardiography, transthoracic, prosthetic cardiac valve disorders, pannus, inspection, hospital admission, gold standard, prostheses

Issue Section: Sunday 1 September 2019 
Published on behalf of the European Society of Cardiology. All rights

reserved. (c) The Author(s) 2019. For permissions, please email:

journals.permissions@oup.com.

This article is published and distributed under the terms of the Oxford University Press, Standard Journals Publication Model (https://academic.oup.com/journals/pages/open_access/funder_policies/chorus/standard_publication_model)

You do not currently have access to this article.

\section{Sign in}

Don't already have an Oxford Academic account? Register

\section{Oxford Academic account}

Email address / Username

Forgot password?

Don't have an account?

\section{European Society of Cardiology members}

OESC

European Society

of Cardiology

Sign in via society site

\section{Sign in via your Institution}

Sign in 


\section{Purchase}

Subscription prices and ordering

\section{Short-term Access}

To purchase short term access, please sign in to your Oxford Academic account above.

Don't already have an Oxford Academic account? Register

P1780

Usefulness of multimodality imaging approach in the diagnosis of mechanical prosthetic valve dysfunction - 24 Hours access

EUR $€ 27.00 \quad$ GBP $£ 20.00 \quad$ USD $\$ 35.00$ 